Review

\title{
Preparation and Application of Iron Oxide Nanoclusters
}

\author{
Angelo J. Antone ${ }^{1}$, Zaicheng Sun ${ }^{2, *(\mathbb{D})}$ and Yuping Bao ${ }^{1,2, *(\mathbb{C})}$ \\ 1 Box 870203, Chemical and Biological Engineering, The University of Alabama, Tuscaloosa, AL 35487, USA \\ 2 Beijing Key Lab for Green Catalysis and Separation, Department of Chemistry and Chemical Engineering, \\ School of Environment and Energy Engineering, Beijing University of Technology, 100 Pingleyuan, Chaoyan, \\ Beijing 100124, China \\ * Correspondence: sunzc@bjut.edu.cn (Z.S.); ybao@eng.ua.edu (Y.B.); Tel.: +001-205-348-9869 (Y.B.)
}

Received: 10 May 2019; Accepted: 25 July 2019; Published: 1 August 2019

check for updates

\begin{abstract}
Magnetic iron oxide nanoclusters, which refers to a group of individual nanoparticles, have recently attracted much attention because of their distinctive behaviors compared to individual nanoparticles. In this review, we discuss preparation methods for creating iron oxide nanoclusters, focusing on synthetic procedures, formation mechanisms, and the quality of the products. Then, we discuss the emerging applications for iron oxide nanoclusters in various fields, covering traditional and novel applications in magnetic separation, bioimaging, drug delivery, and magnetically responsive photonic crystals.
\end{abstract}

Keywords: iron oxide nanoclusters; superparticles; magnetically responsive photonic crystals; collective behaviors; magnetic separation; bioimaging

\section{Introduction}

Magnetic iron oxide nanoclusters, which refers to a group of individual nanoparticles, have recently attracted much attention because of their distinctive behaviors compared to individual nanoparticles [1-3]. The magnetic properties of iron oxide nanoparticles are strongly dependent on size, yielding single-domain regimes and a superparamagnetic limit [4]. Because of the superparamagnetic limit, iron oxide nanoparticles with grain sizes above $25 \mathrm{~nm}$ (depending on crystal phases (magnetite or maghemite)) are generally ferromagnetic at room temperature. The magnetic interactions between nanoparticles lead to aggregation in solution, which limits their uses in certain applications, such as drug delivery. The superparamagnetic limit also sets a threshold for the maximum moment to be reached. The formation of nanoclusters by assembling individual iron oxide nanoparticles has the potential to overcome this limitation by increasing magnetic moments while at the same time maintaining superparamagnetic behaviors [5].

Magnetic iron oxide nanoclusters combine the properties of individual nanoparticles and exhibit collective behaviors due to interactions between individual nanoparticles [3]. In addition, the collective behaviors of these nanoclusters can be controlled by tuning the size and shape of individual nanoparticles, the interspacing between nanoparticles, and the properties of the capping molecules of individual nanoparticles $[2,3,6]$. Particularly, magnetic nanoclusters can be manipulated with applied magnetic fields, leading to novel functional materials. Iron oxide nanoclusters have great potential to improve the performance of individual nanoparticles and develop advanced materials with novel functions.

This review will discuss the preparation methods of iron oxide nanoclusters and their applications in various areas. For preparation methods, the discussion will focus on synthetic procedures, formation mechanisms, and the quality of nanoclusters in terms of size distribution, size control, and scalability. 
On the other hand, the application discussion will cover the use of improved magnetic properties and novel applications that have been recently developed, such as cell membrane-encapsulated iron oxide nanoclusters for drug screening, drug delivery, and tumor targeting.

\section{Preparation of Iron Oxide Nanoclusters}

Magnetic iron oxide nanoclusters can be produced either through controlled aggregation of small iron oxide nanoparticles during synthesis (e.g., the polyol method [5]) or the assembly of ligand-capped nanoparticles after synthesis (e.g., solvophobic interactions [7]). Each of these methods has its advantages and disadvantages, which will be discussed in the following section.

\subsection{Controlled Aggregation of Nanoparticles during Synthesis}

For controlled aggregation methods, small-sized iron oxide nanoparticles $(<10 \mathrm{~nm})$ are first formed in a supersaturated solution of iron precursors through nucleation and growth, and then these small-sized nanoparticles spontaneously aggregate into larger nanoclusters $(50-300 \mathrm{~nm})$ in a single step. The grain (small nanoparticle) size and final nanocluster size can be controlled by adjusting reaction conditions. Several controlled aggregation methods have been developed to produce iron oxide nanoclusters in a single step, such as the polyol method, solvothermal synthesis, thermal decomposition, and microwave methods.

\subsubsection{Polyol Method}

The polyol method developed by Yin et al. involves the injection of iron salts (e.g., iron chloride) into a polyol solution (e.g., diethylene glycol) at a high temperature $\left(>200{ }^{\circ} \mathrm{C}\right)$ in the presence of capping molecules (e.g., polyacrylic acid) under basic conditions $[5,8]$. This method produces highly water-soluble and monodisperse iron oxide nanoclusters (30-200 $\mathrm{nm}$ ) in a single step $[2,5,8,9]$. The grain size and nanocluster size are controlled by the amounts of base injected into the reaction [5].

Figure 1a-d shows representative transmission electron microscopy (TEM) images of differently sized iron oxide nanoclusters from the polyol method, where the nanocluster sizes were controlled by the amounts of sodium hydroxide ethylene glycol solution. These nanoclusters consisted of a number of small-sized iron oxide nanoparticles $(<10 \mathrm{~nm})$ yielding superparamagnetic behaviors at room temperature but with enhanced saturation magnetization (Figure 1e). In a similar study, control of both the grain size and nanocluster size was achieved by adjusting the concentration and injection speed of the base solution [6]. Because of the polyacrylic acid coatings, iron oxide nanoclusters from this method are highly negatively charged and well dispersed in aqueous solution. These nanoclusters can be directly used for various applications, such as magnetically responsive photonic crystals $[6,9]$. 

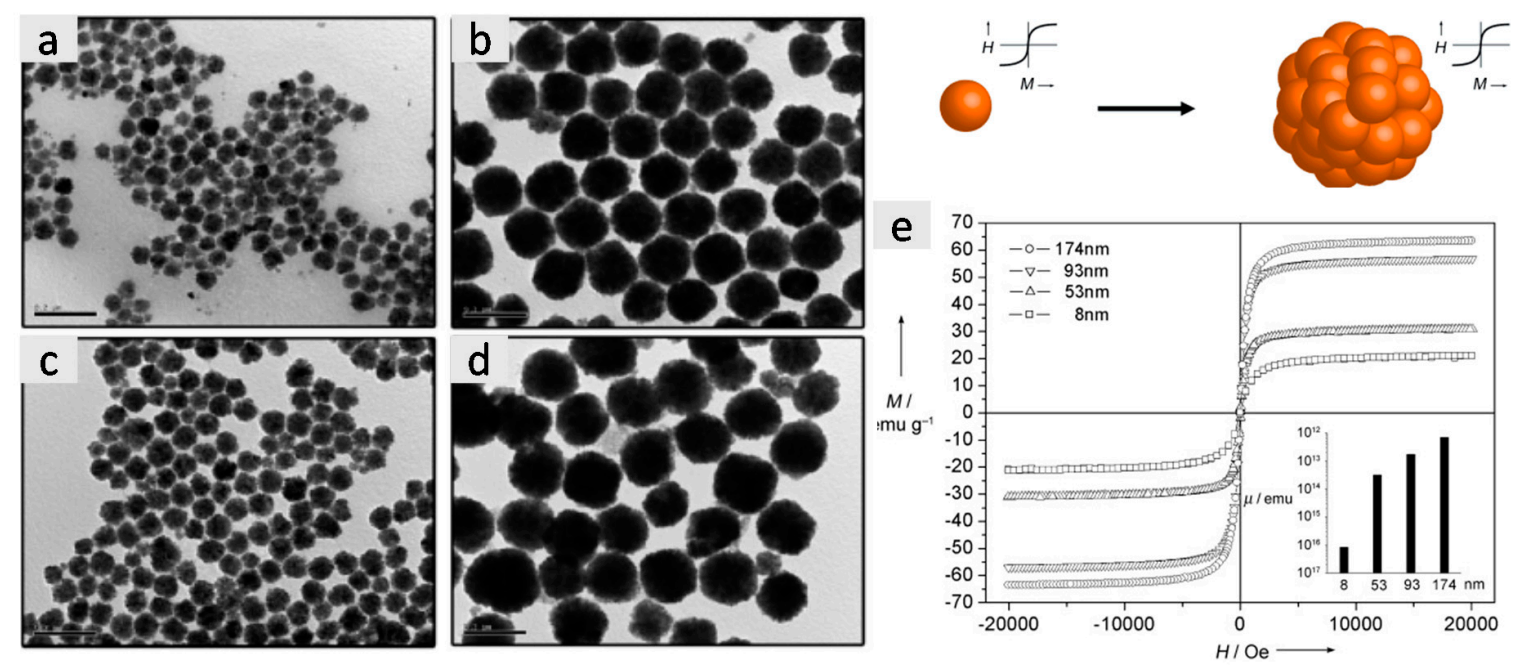

Figure 1. Iron oxide nanoclusters produced with the polyol method: (a-d) representative TEM images demonstrating size control, scale bar $200 \mathrm{~nm}$; (e) magnetization versus applied field curves, suggesting superparamagnetic behaviors but with increased magnetic moments for larger nanoclusters. (Adapted with permission from Reference [5]).

\subsubsection{Solvothermal Synthesis}

Solvothermal synthesis involves first mixing reactants (e.g., iron chloride, sodium acetate, capping molecules) in reducing solvents (e.g., ethylene glycol or diethylene glycol) under stirring: Then the mixture reacts in a sealed Teflon-lined stainless steel hydrothermal reactor at a high temperature $\left(>200^{\circ} \mathrm{C}\right)$ to induce iron oxide nanocluster formation [10-14]. This method is highly attractive for several reasons: first, the capping molecules can be selected from a variety of organic acids for different surface chemistries, such as sodium citrate [14], polyacrylic acids [15], and 5-sulfosalicylic acid [13]; and second, the grain and nanocluster size can be controlled easily by adjusting the ratios and concentrations of the reactants [10]. In addition, porous iron oxide nanoclusters can be generated by simply using gas-forming reactants, such as ammonium acetate [16]. Most importantly, the scalable production of the synthetic process (up to $200 \mathrm{~g}$ per batch) has been demonstrated without the quality of the iron oxide nanoclusters being affected [14]. Figure 2 shows synthetic procedure, formation mechanism, representative scanning electron microscopy (SEM), and TEM images of iron oxide nanoclusters from gram-scale solvothermal synthesis. 


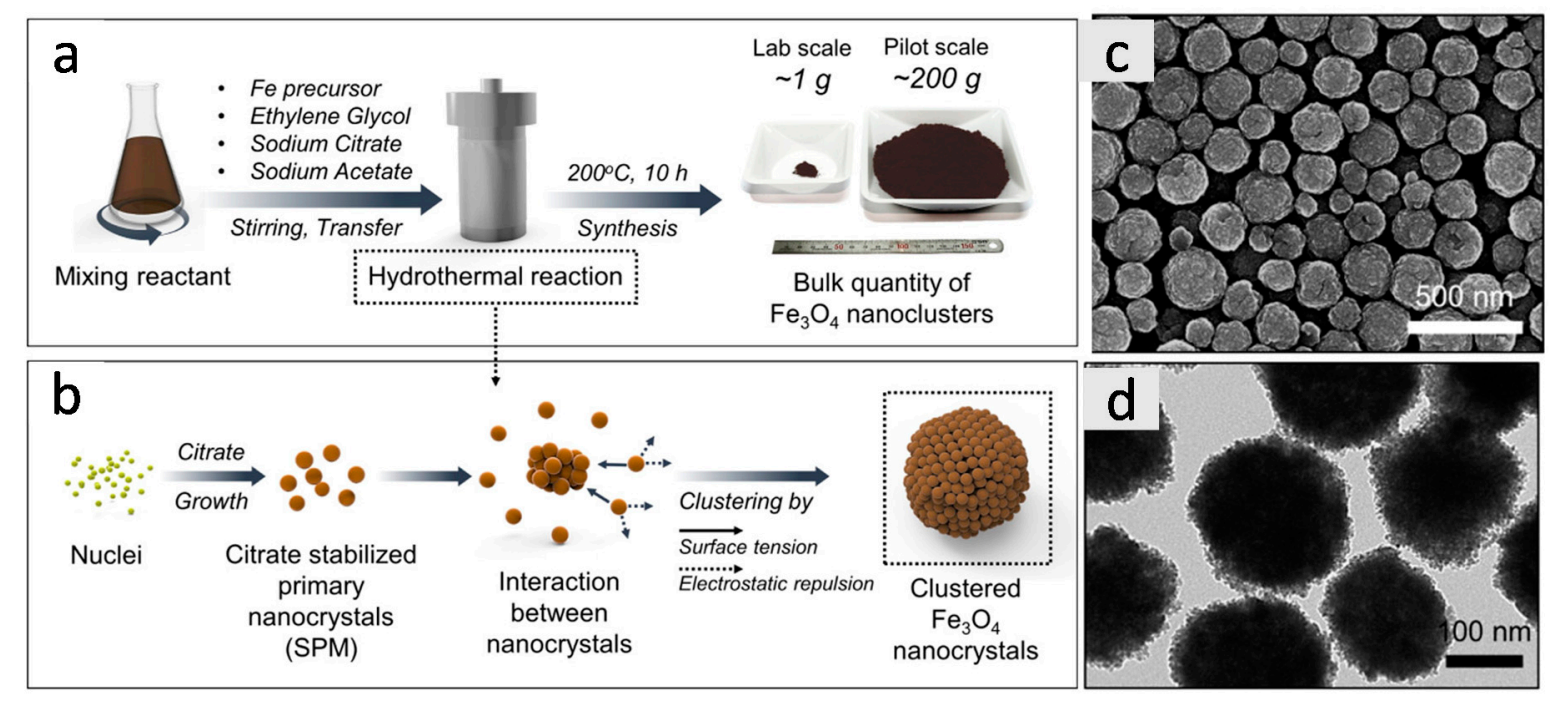

Figure 2. Gram-scale solvothermal synthesis of iron oxide nanoclusters: (a) schematic illustration of the procedure; (b) proposed mechanism of the nanocluster formation; (c,d) SEM and TEM images indicating the quality of the nanoclusters (adapted from Reference [14] with permission; copyright American Chemical Society, 2018).

The nanoclusters generated from the solvothermal synthesis are highly soluble in aqueous solution and can be directly used for various applications [14]. Porous iron oxide nanoclusters can also be produced through slight modifications of the process by adding gas forming reagents, and these porous nanoclusters have been explored for magnetically responsive drug delivery with high drug loading capacity [16].

In addition to the polyol method and solvothermal synthesis, other synthetic methods have been explored for the production of iron oxide nanoclusters in a single step, such as thermal decomposition [17] and microwave irritation [18]. However, the quality of the nanoclusters has not been comparable to these two methods. In particular, the size distribution of the nanoclusters from these two methods is much wider.

\subsection{Controlled Assembly of Ligand-Capped Nanoparticles}

The formation of nanoclusters from the controlled assembly of ligand-capped nanoparticles involves two steps: the synthesis of monodisperse ligand-capped iron oxide nanoparticles $(10-20 \mathrm{~nm})$ and the controlled assembly of nanoparticles under specific processing conditions. The processing conditions trigger the assembly process and affect the quality of the final products, such as ligand etching [19] and solvophobic interaction [7,20].

\subsubsection{Ligand Etching}

Nanoparticles are generally coated with a layer of ligands to prevent them from aggregation. The ligand etching process involves replacing the original capping molecules with weakly bound ligands, which causes the destabilization of nanoparticles and subsequent aggregation and nanocluster formation (Figure 3a). The size and shape of the assembled secondary structures are dependent on the ligands used for the striping process. For example, the addition of diol molecules into the solution of oleic acid-coated iron oxide nanoparticles $(\sim 13 \mathrm{~nm})$ led to nanoparticle destabilization and subsequent secondary structure formation [19]. Depending on the types of diol molecules, dimers, oligomers, and nanoclusters were formed during the ligand stripping process. Figure $3 \mathrm{~b}-\mathrm{e}$ shows representative TEM images of the secondary structures with the addition of different diol molecules. 


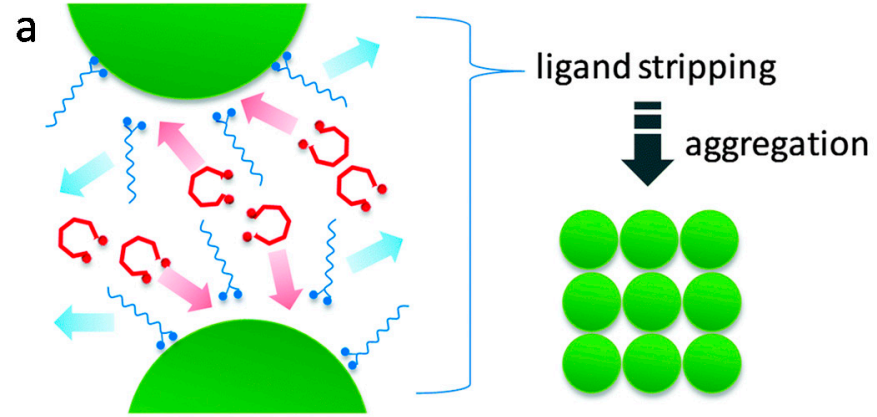

Schematic illustration of the "ligand stripping" method.

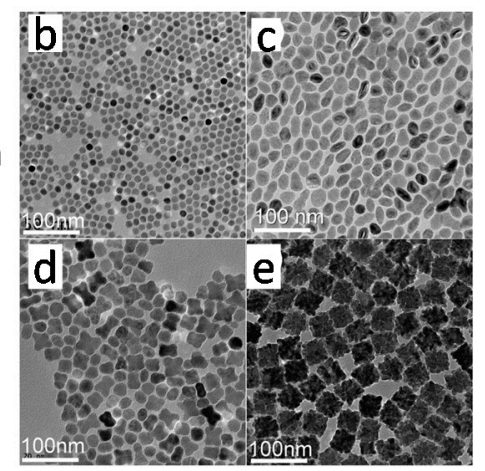

Figure 3. Nanocluster formation via the ligand stripping method: (a) illustration of the ligand stripping method process, (b) spherical nanoparticles with no diol addition, (c) dimer formation induced by the addition of polyethylene glycol 400, (d) oligomer induced by the addition of triethylene glycol, and (e) nanocluster induced by the addition of diethylene glycol (adapted from Reference [19]; reproduced with permission from the Royal Society of Chemistry).

In a similar study, magnetic iron oxide nanoclusters were prepared using competitive stabilizer desorption, where oleic acid-coated iron oxide nanoparticles were mixed with cyanopropyl-modified silica nanoparticles. The silica particles competed for capping ligands on the iron oxide nanoparticle surfaces, which caused the destabilization of iron oxide nanoparticles and the subsequent formation of magnetic nanoclusters [21]. Compared to the single-step aggregation methods, the nanocluster sizes from ligand stripping are smaller and mainly soluble in organic solvents because of the presence of hydrophobic ligands.

\subsubsection{Solvophobic Interactions}

The solvophobic interaction method involves mixing hydrophobic ligand-coated (e.g., oleic acid) iron oxide nanoparticles with surfactants (e.g., dodecyltrimethylammonium bromide, DTAB) to form micelle structures. After evaporating away the organic solvent, a group of iron oxide nanoparticles are combined within the micelles. Subsequently, the micelle solution goes through an annealing process in ethylene glycol in the presence of capping molecules at an elevated temperature (e.g., $80^{\circ} \mathrm{C}$ ), leading to nanocluster formation $[7,20]$. Figure 4 a illustrates the preparation process via solvophobic interactions. The size of the nanoclusters can be controlled by the relative ratios of nanoparticles to surfactants, and the stability of the nanoclusters is affected by the structures of surfactants and capping molecules. Figure $4 b, c$ shows representative TEM and SEM images of iron oxide nanoclusters formed from 6-nm oleic acid-iron oxide nanoparticles using DTAB as a micelle-forming agent and poly(vinylpyrrolidone) as capping molecules. The main advantage of this method is that it is not limited to iron oxide nanoparticles, but can be easily applied to any other type of nanoparticle with a hydrophobic surface coating. 


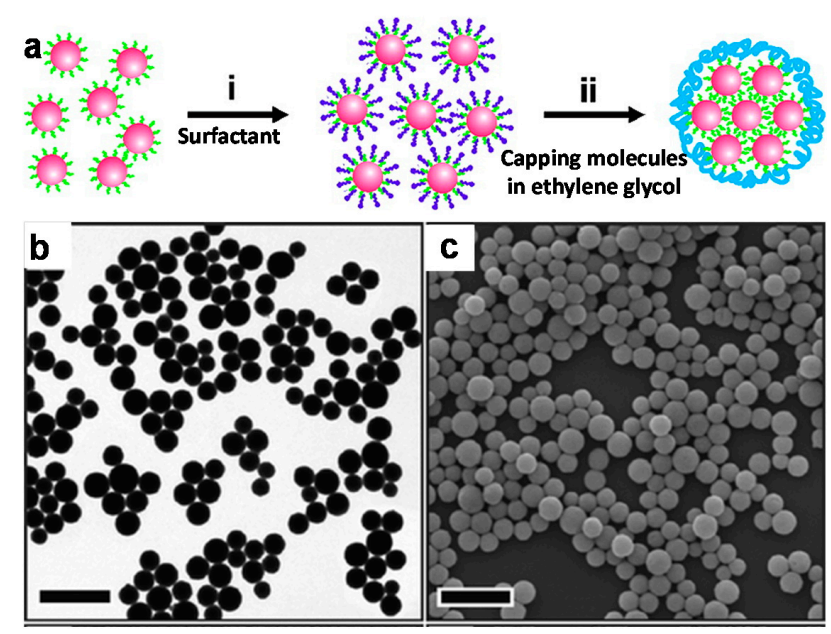

Figure 4. Iron oxide nanoclusters via solvophobic interactions: (a) scheme of the formation process, (b) TEM image, and (c) SEM image, scale bar $500 \mathrm{~nm}$ (adapted from Reference [20], with permission; copyright American Chemical Society, 2007).

\subsection{Matrix Encapsulation of Nanoparticles}

For the matrix encapsulation method, nanocluster formation is assisted by the matrices, where iron oxide nanoparticles are mixed with the selected matrix and the induced matrix crosslinking leads to the formation of nanoclusters. Several types of matrices have been reported to fabricate iron oxide nanoclusters, such as proteins [22], polymers [23-25], silica [26], etc. The control of the aggregation process and the quality of the nanoclusters are highly specific to the choice of matrices. For example, protein encapsulation of iron oxide nanoparticles is normally induced by ethanol addition followed by surface crosslinking with glutaraldehyde [22]. In contrast, polydopamine encapsulation can be easily triggered by changing the $\mathrm{pH}$ of the solution [23]. In addition, the salt concentration, amount and addition speed of ethanol, and protein concentration all affect the quality of bovine serum albumin (BSA) encapsulated in ultrasmall iron oxide nanoparticles [22].

Figure 5 shows representative TEM images of iron oxide nanoclusters that were produced with different matrices, where the polymer shells can be clearly seen (Figure $5 \mathrm{a}$ ), but the silica and protein encapsulation formed matrix-iron oxide composite materials (Figure $5 b, c$ ). The matrix-assisted method has several distinctive advantages: first, drug molecules can be simultaneously encapsulated into the nanoclusters during the aggregation process, creating magnetic resonance imaging (MRI)-visible drug delivery vehicles; second, biocompatibility and water solubility can be easily achieved based on the choices of the matrices; and finally, by tuning the nanocluster sizes, other functionality can be achieved, such as ultrasound response [27].
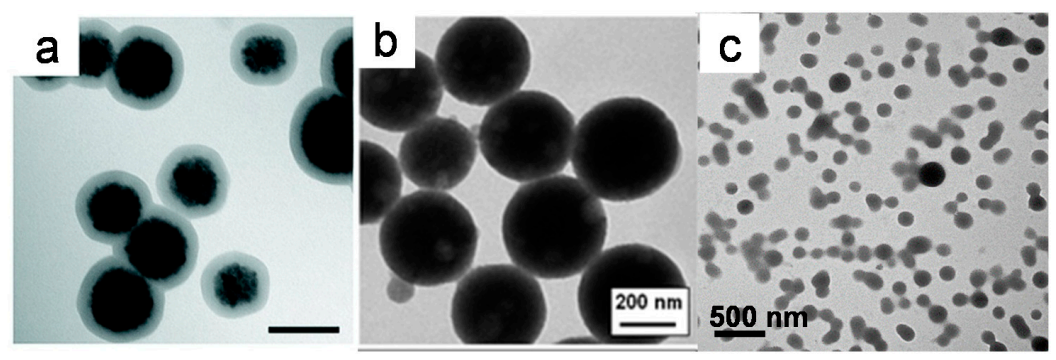

Figure 5. TEM images of matrix-encapsulated iron oxide nanoparticles: (a) hydrogel, (b) silica, and (c) bovine serum albumin protein ((a) is adapted from Reference [24] (with permission), copyright American Chemical Society, 2011; (b) is adapted from Reference [26] (with permission), copyright American Chemical Society, 2008; (c) is adapted from Reference [22], reproduced with permission from the Royal Society of Chemistry). 


\section{Applications of Iron Oxide Nanoclusters}

Iron oxide nanoclusters have been explored for numerous applications [3,28], including rapid magnetic separation [29], MRI contract agents with enhanced sensitivity [30], nanocarriers with high drug loading capacity [16], and magnetically responsive photonic crystals [6,9,31]. The following section will discuss these applications of magnetic nanoclusters in detail to present their potentials as functional materials with improved performance.

\subsection{Iron Oxide Nanoclusters for Magnetic Separation}

Magnetic separation is the most traditional use for magnetic nanoparticles and utilizes the large surface areas of nanoparticles to enhance adsorption capacity. Subsequently, magnetic fields are applied to extract, enrich, or separate compounds of interest [32-34]. During magnetic separation, the nanoparticles have to overcome the drag forces in solution: therefore, the higher the magnetic moments of nanoparticles, the faster the separation processes. The formation of magnetic nanoclusters increases the magnetic moments, leading to the fast response of separation processes. However, the size increase of nanoclusters causes decreases in the total surface area of nanoclusters per given mass. Therefore, an optimal size range of nanoclusters for magnetic separation needs to be considered for efficient separation and large adsorption capacity. Several nanocluster systems have been designed for the separation, enrichment, and detection of biomolecules [35-37], organisms [38,39], or inorganic ions $[40,41]$.

For example, antibody-functionalized iron oxide nanospheres $(\sim 400 \mathrm{~nm})$ (through the assembly of iron oxide nanoparticles onto copolymers) have been used for the quick enrichment of bacteria [38]. The nanospheres showed a fast magnetic response of less than one minute and an over-96\% capture efficiency of bacteria at ultralow concentrations ( $<50$ colony-forming unit (CFU)/mL) [38]. Kim et al. have shown the highly selective detection and rapid separation of pathogenic organisms using magnetic iron oxide nanoclusters [39]. In that study, the iron oxide nanoclusters were prepared through solvophobic interactions using polysorbate 80 as a micelle surfactant (Figure 6a). Then, a monoclonal antibody was conjugated on the nanocluster surface for pathogen binding. The magnetic properties of iron oxide nanoclusters were optimized theoretically by calculating size-dependent magnetic forces and Brownian forces of nanoclusters, suggesting that nanoclusters of about $200 \mathrm{~nm}$ provided efficient separation and large separation capacity (Figure 6b) [39]. Figure $6 \mathrm{c}$ shows the detection principle and the nanoclusters binding to the pathogens via two different binding sites ( $\mathrm{H}$ and $\mathrm{O}$ antigens).

Most recently, we [42] and others [43,44] have developed a new type of magnetic separation method based on cell membrane-encapsulated iron oxide nanoclusters. Compared to traditional magnetic separation techniques using immobilized ligands on nanocluster surfaces to bind the targets, the new technique uses functional transmembrane receptors as binding sites to identify the targets. The complete embedment of iron oxide nanoclusters inside cell membranes overcame the nonspecific binding problems because magnetic nanoclusters were not in direct contact with the analyte solution. Figure 7a illustrates the design of the cell membrane-encapsulated nanoclusters. The choice of the cell membrane depends on the specific targets to be extracted. 

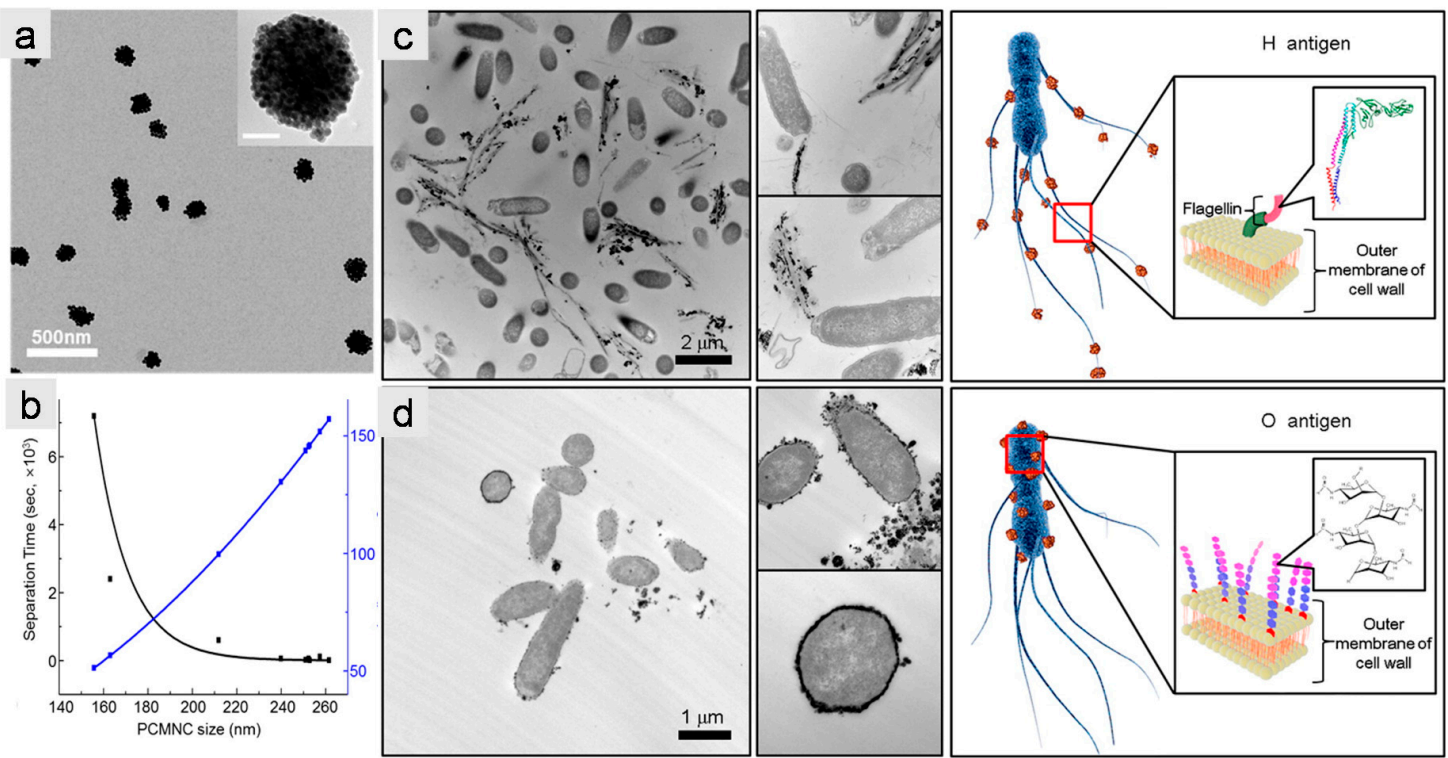

Figure 6. Selective detention of pathogens using iron oxide nanoclusters: (a) TEM images of nanoclusters, (b) relationship between magnetic separation time (black line) and magnetic force under specific field gradients (blue line), (c) H-antigen-specific binding of nanoclusters to flagella, and (d) O-antigen-specific binding of the nanoclusters on the surface of the cell body (adapted from Reference [39], with permission; copyright American Chemical Society, 2016).

For example, in order to extract nicotine molecules from tobacco smoke condensates, we created cell membrane-encapsulated nanoclusters using human cell line overexpressing $\alpha_{3} \beta_{4}$ receptors, which bind to nicotine molecules specifically. Figure $7 \mathrm{~b}$ shows representative TEM images of the iron oxide nanoclusters prepared using cell membranes with $\alpha_{3} \beta_{4}$ nicotinic receptors. Even though the cell receptors were not visible on the TEM images of the cell membrane-encapsulated iron oxide nanoclusters, the fishing experiments clearly demonstrated binding specificity and efficiency. The nicotine receptors on the surfaces were able to fish out the nicotine molecules from tobacco smoke condensates, and all other compounds without specific binding to the nicotine receptors were washed out, as shown in the washing and elution chromatograms of the high-performance liquid chromatography (HPLC) (Figure 7c). In addition, iron oxide nanoclusters coated with cell membranes without nicotine receptors showed no binding to nicotine in the smoke condensates, suggesting specific binding between nicotine and $\alpha_{3} \beta_{4}$ receptors. This new magnetic separation will greatly benefit the discovery of new drug candidate targeting transmembrane receptors. Most importantly, this technique can be easily applied to any other transmembrane receptors.
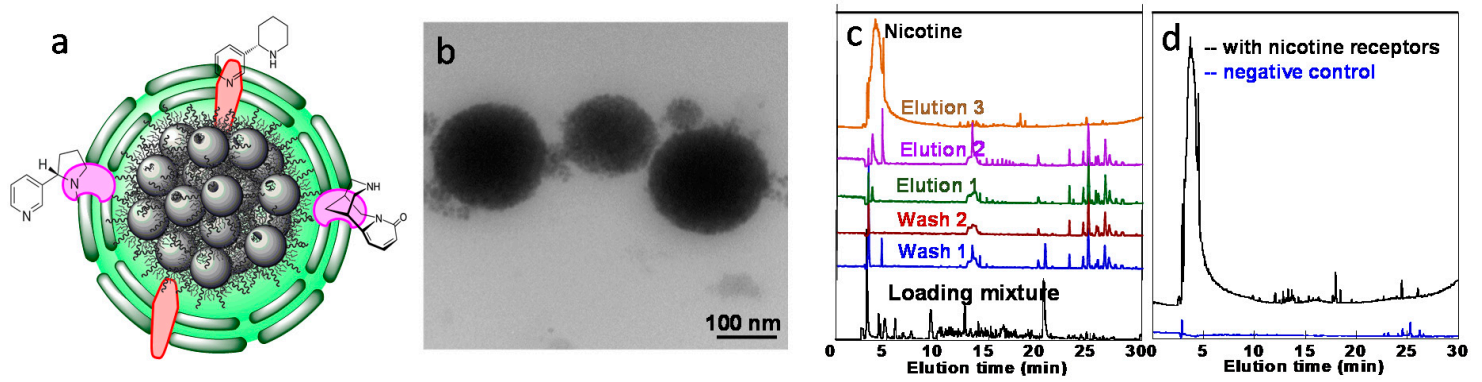

Figure 7. Cell membrane-encapsulated iron oxide nanoclusters: (a) design concept, (b) TEM image, (c) HPLC washing and elution chromatograms of fishing experiments using $\alpha_{3} \beta_{4}$ receptors from smoke condensates, (d) comparison of elution profiles with and without $\alpha_{3} \beta_{4}$ receptors (adapted from Reference [42]; reproduced with permission from the Royal Society of Chemistry). 
In a similar study, iron oxide nanoparticles were encapsulated inside red blood cell membranes for virus targeting and isolation [45]. The cell membranes were modified with sialic acid molecules, which formed stable clusters with influenza viruses. The encapsulated superparamagnetic iron oxide nanoparticles enabled the quick enrichment of the influenza virus via magnetic extraction. The enriched viral samples significantly enhanced virus detection through multiple viral quantification methods, such as the immunochromatographic strip test and cell-based tittering assays.

Additionally, iron oxide nanoclusters have been applied to the enhanced removal of molybdate from surface water [40], the reduction of arsenic concentrations below the World Health Organization (WHO) permissible safety limit for drinking water [41], enrichments of chemical molecules for analysis [46], and protein adsorption [37,47].

\subsection{Biomedical Applications of Iron Oxide Nanoclusters}

The biomedical applications of iron oxide nanoclusters have been focused on magnetically triggered drug release [48-51] and MRI contrast agents with high sensitivity [30,52-54]. For magnetically triggered drug release, either iron oxide nanoparticles $(>10 \mathrm{~nm})$ and drugs were colocalized in nanocarriers [55] or porous iron oxide nanoclusters were created to increase drug loading by surface adsorption [16]. Under alternating magnetic fields (AMFs), local heat was generated from iron oxide nanoparticles, which elevated the local temperatures and subsequently caused drug release.

For example, iron oxide nanoparticle-loaded microcapsules were prepared through layer-by-layer deposition of positively and negatively charged polyelectrolytes onto a calcium carbonate template. By replacing the negatively charged electrolyte with negatively charged nanoparticles, the nanoparticles were incorporated inside the shell, as shown in Figure 8a. Figure 8b shows a representative TEM image of a capsule, where the darkness of the shell indicates the successful encapsulation of iron oxide nanoparticles. The drug molecules were loaded inside the capsule after leaking out of the template. Under AMFs, local heat was generated from the nanoparticles inside the shell, which triggered drug release. Compared to samples without applying AMFs, the drug release was significantly enhanced after applying $90 \mathrm{~min}$ of AMFs ( $300 \mathrm{kHz}$ and $24 \mathrm{kAm}^{-1}$ ), as shown in Figure 8c. Compared to drug release triggered by photothermal stimulation, magnetic fields have better tissue penetration. In addition, the localization of iron oxide nanoparticles inside the shells decreased the permeability of microcapsules, preventing premature drug release before applying external stimuli [27].

Without matrix assistance, iron oxide nanoclusters are generally made into porous structures for drug delivery applications. The high surface area and cavities of the porous structures increase surface drug adsorption, leading to enhanced drug loading [16,56]. For example, porous iron oxide nanoclusters were prepared using solvothermal synthesis, where sodium acetate was used to create the porous structure because of ammonia gas bubble formation during synthesis [16]. Figure $8 \mathrm{~d}$ shows an illustration of porous nanoclusters, and Figure 8e shows a representative TEM image of porous iron oxide nanoparticles. These as-prepared porous iron oxide nanoclusters served as great nanocarriers for hydrophobic drugs, with a demonstrated loading capacity as high as $35.0 \mathrm{wt} \%$ for paclitaxel (Figure 8f). The antitumor efficacy of paclitaxel-loaded nanoclusters under AFMs was significantly enhanced compared to free drugs. 

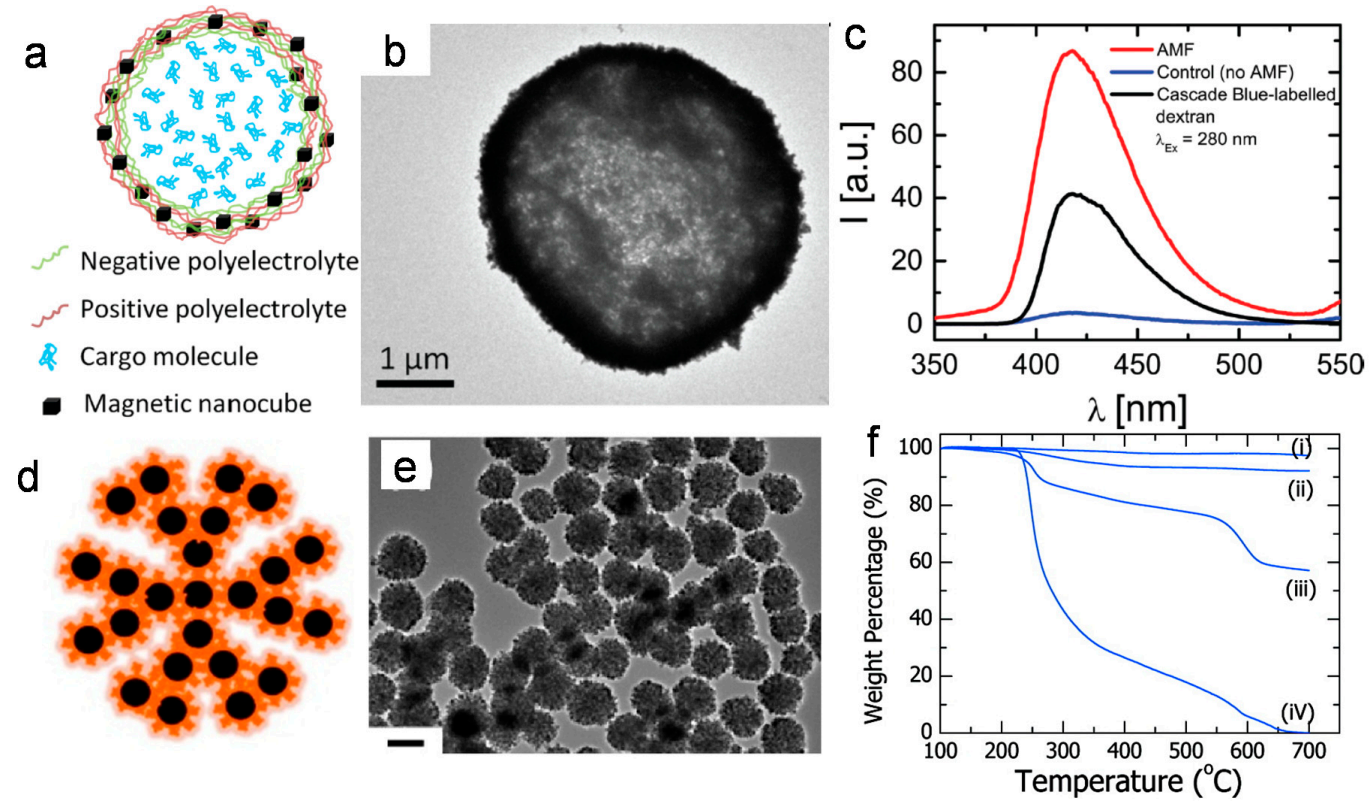

Figure 8. Iron oxide nanoclusters for drug delivery: $(\mathbf{a}-\mathbf{c})$ design, TEM image, and magnetically triggered drug release profile of iron oxide-decorated microcapsules; and (d-f) illustration, TEM image, and thermogravimetric analysis plots representing the drug loading capacity of porous iron oxide nanoclusters $(\mathbf{a}-\mathbf{c})$ are adapted from Reference [55], reproduced with permission from the Royal Society of Chemistry; (d-f) are adapted from Reference [16] (with permission), copyright American Chemical Society, 2011).

Recently, cell membrane-coated iron oxide nanoparticles have been created for tumor targeting and drug delivery [57-60]. For example, macrophage membrane-coated iron oxide nanoparticles have been shown to be effective nanocarriers for tumor targeting and therapy [58]. In that study, the functional transmembrane receptors were able to recognize cancer cells via cell-cell adhesion between macrophage and cancer cell surfaces for effective cell targeting. The encapsulated iron oxide nanoparticles were used as photoabsorbing agents for photothermal therapy. Similar concepts have also been demonstrated for myeloid-derived suppressor cell membrane-coated magnetic nanoparticles, which performed well in immune evasion, active tumor targeting, and photothermal therapy-induced tumor killing [59].

For MRI applications, iron oxide nanoclusters have been explored as both $\mathrm{T}_{1}$ (positive) and $\mathrm{T}_{2}$ (negative) contrast agents. The iron oxide-based $\mathrm{T}_{1}$ contrast agents were mainly either ultrasmall $(<4 \mathrm{~nm})$ nanoparticles $[22,61,62]$ or ultrathin (diameter $<4 \mathrm{~nm}$ ) nanowires [63,64]. Nanocluster formation was mainly to overcome the short blood circulation time of these nanostructures due to their small dimensions [22]. For instance, tannic acid-coated ultrasmall iron oxide nanoparticles $(3 \sim 4 \mathrm{~nm})$ only had about $15 \mathrm{~min}$ of blood circulation time and were quickly cleared by the renal system [22]. Encapsulating these ultrasmall iron oxide nanoparticles inside BSA nanoclusters exhibited enhanced $\mathrm{T}_{1}$ permanence with higher $\mathrm{r}_{1}$ relaxivity and increased blood circulation times [22]. Figure 9a shows a comparison between phantom MRI images of free nanoparticles and BSA ultrasmall iron oxide nanoclusters, suggesting great $T_{1}$ brightening of the nanoclusters. Figure $9 \mathrm{~b}$ shows MRI images of mice before and $2 \mathrm{~h}$ post-injection with BSA ultrasmall iron oxide nanoclusters, where the brain and kidney regions were significantly brightened, suggesting the nanoclusters remained in the blood stream even $2 \mathrm{~h}$ after injection. Similarly, ultrasmall iron oxide nanoparticles sandwiched between polymer layers of layer-by-layer assembled microcapsules showed not only increased blood circulation time, but also served as great drug nanocarriers for ultrasound-triggered drug release [27]. Figure $9 \mathrm{c}$ shows MRI images of a control mouse and a mouse $48 \mathrm{~h}$ post-injection, where the bright region near the heart suggested the long blood circulation time of these nanoparticle-loaded capsules. Because of their larger sizes, these capsules were sensitive to ultrasound, which enhanced drug localization at the tumor site with ultrasound treatment (Figure 9d). 
a

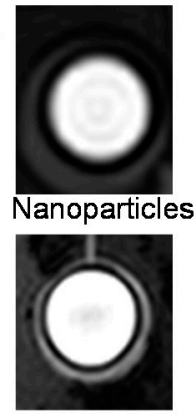

BSA Nanoclusters
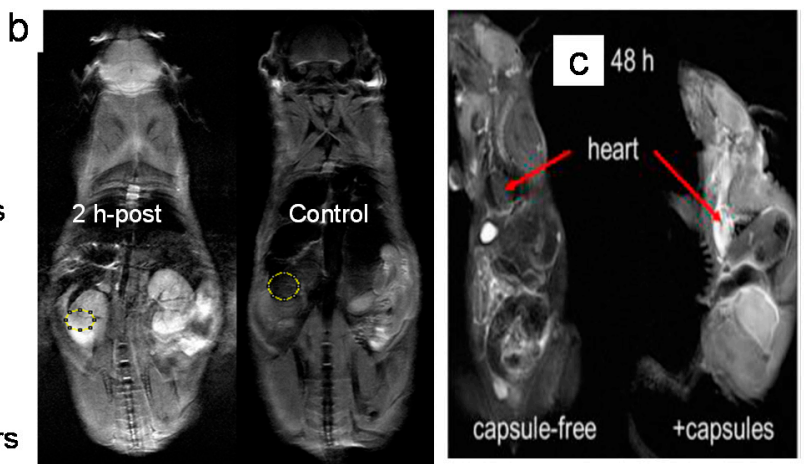

Figure 9. (a) Phantom MRI images of free ultrasmall nanoparticles and bovine serum albumin (BSA) ultrasmall iron oxide nanoclusters, (b) MRI images of mice before and $2 \mathrm{~h}$ post-injection with BSA ultrasmall iron oxide nanoclusters, (c) MRI images of a mouse $48 \mathrm{~h}$ after injection with ultrasmall iron oxide nanoparticle-loaded capsules (right) and a control (left), and (d) iron quantification showing the increased accumulation of an ultrasound-treated tumor after capsule injection $((\mathbf{a}, \mathbf{b})$ are adapted from Reference [22], reproduced with permission from the Royal Society of Chemistry; (c,d) are adapted from Reference [27]).

$\mathrm{T}_{2}$ MRI contrast agents are mainly superparamagnetic nanoparticles, and nanocluster formation increases the magnetic signal and subsequently enhances the imaging sensitivity or cell labeling efficiency $[24,30,52,53,56,65-67]$. For example, differently sized iron oxide nanoclusters via solvothermal synthesis showed higher $\mathrm{r}_{2}$ relaxivity for nanocluster sizes of around $50-60 \mathrm{~nm}$. The $\mathrm{r}_{2}$ relaxivity of 63-nm iron oxide nanoclusters was more than three times higher than that of commercial products (Resovist) [30]. In addition, the cellular take efficiency of 63-nm iron oxide nanoclusters by macrophage cells was 10 times higher than that of Resovist [30]. Enhanced MRI sensitivity and increased cellular uptake make iron oxide nanoclusters great candidates for cellular MRI.

\subsection{Optical Applications of Iron Oxide Nanoclusters}

Iron oxide nanoclusters synthesized by the polyol method are negatively charged and highly water soluble. Under magnetic fields, these nanoclusters assemble into chain-like structures and diffract visible light. Therefore, these nanoclusters are used as building blocks to develop magnetically controlled photonic crystals $[6,9,31]$. The color of diffracted light can be tuned through adjustments to nanocluster size, interspacing between nanoclusters, and the strength of the applied magnetic fields. Using superparamagnetic polyacrylic acid-coated iron oxide nanoclusters of $120 \mathrm{~nm}$ as an example (Figure 10a-c), it was demonstrated that the color of the diffracted lights shifted from blue to red when the applied magnetic fields increased (Figure 10d). The reflection spectra clearly showed the dependence of the refracted light wavelength on the strength of the applied magnetic field, where the field strength was tuned by changing the distance between the magnet and the nanoparticle solution (Figure 10e) [5]. In addition, the optical response of the colloidal photonic crystals to the changes in the external magnetic field was fully reversible. 

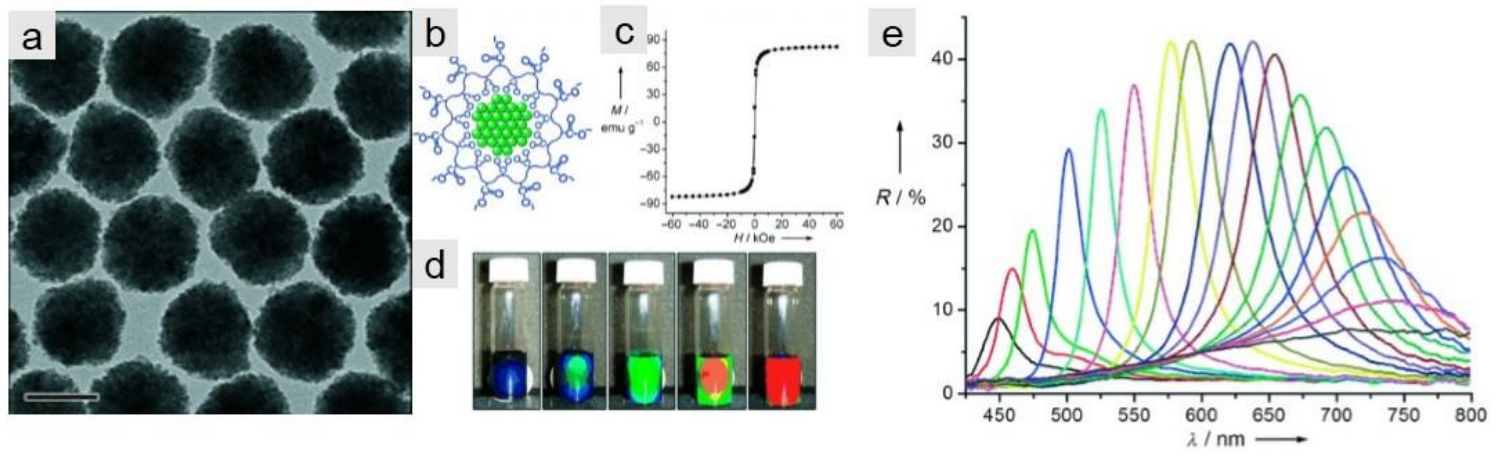

Figure 10. Iron oxide nanoclusters as building blocks for photonic crystals: (a) TEM image of iron oxide nanoclusters, scale bar $100 \mathrm{~nm}$; (b) schematic illustration of polyacrylate coating on nanocluster surfaces; (c) superparamagnetic behavior of nanoclusters at room temperature; (d) photographs of photonic crystals formed in response to an external magnetic field; and (e) UV-Vis reflectance spectra (adapted with permission from Reference [9]).

In a similar study [6], iron oxide nanoclusters of different cluster and grain sizes were tested for optical response. It was discovered that a critical nanocluster size $(\sim 40 \mathrm{~nm})$ existed, below which no changes were observed in light diffraction with applied magnetic fields. In addition, larger nanoclusters $(>160 \mathrm{~nm})$ preferably diffracted red light in relatively low magnetic fields, while the smaller nanoclusters $(<100 \mathrm{~nm})$ diffracted blue light in stronger magnetic fields.

\section{Summary and Outlook}

In summary, we have discussed the preparation methods and emerging applications of iron oxide nanoclusters. Compared to individual iron oxide nanoparticles, these nanoclusters are much larger in size (hundreds $\mathrm{nm}$ ), but remain superparamagnetic. Several synthetic methods have been developed by either directly preparing nanoclusters in a single step (e.g., the polyol method, thermal decomposition, and solvothermal synthesis) or assembling presynthesized ligand-capped nanoparticles under controlled conditions (e.g., solvophobic interaction, ligand etching, and matrix-assisted aggregation). Among the various synthetic methods, solvothermal synthesis is highly attractive, as it produces water-soluble, monodisperse superparamagnetic nanoclusters in a single step. In particular, large-scale production (up to $200 \mathrm{~g}$ per batch) has been demonstrated without scarifying the quality of the product. The enhanced magnetic properties and collective behaviors of these nanoclusters have led to a number of emerging applications, such as rapid magnetic separation and magnetically triggered drug delivery. Of all of these discussed applications, cell membrane-encapsulated nanoclusters are particularly attractive because of their enhanced performance. For example, cell membrane-encapsulated nanoclusters for magnetic separation can overcome the nonspecific binding problems associated with current magnetic bead technologies. The functional transmembrane receptors allow for the specific identification and extraction of targets binding to cell receptors. Depending on the choices of cell membranes, cell membrane-encapsulated nanoclusters are also used for drug delivery and tumor targeting. These biomimetic nanocarriers exhibit much better efficiency compared to traditional nanocarriers.

Funding: This work was funded in part by National Science Foundation (NSF) DMR1149931. Z.S. is funded by Beijing Municipal High Level Innovative Team Building Program (IDHT20180504) and the National Natural Science Foundation of China (21805004, 21671011, 21872001 and 51801006).

Acknowledgments: National Science Foundation (NSF) DMR1149931, Beijing Municipal High Level Innovative Team Building Program (IDHT20180504) and the National Natural Science Foundation of China (21805004, 21671011, 21872001 and 51801006).

Conflicts of Interest: The authors declare no conflicts of interest. 


\section{References}

1. Lee, J.S.; Cha, J.M.; Yoon, H.Y.; Lee, J.K.; Kim, Y.K. Magnetic multi-granule nanoclusters: A model system that exhibits universal size effect of magnetic coercivity. Sci. Rep. 2015, 5, 12135. [CrossRef]

2. Lu, Z.; Yin, Y. Colloidal nanoparticle clusters: Functional materials by design. Chem. Soc. Rev. 2012, 41, 6874-6887. [CrossRef] [PubMed]

3. Kostopoulou, A.; Lappas, A. Colloidal magnetic nanocrystal clusters: Variable length-scale interaction mechanisms, synergetic functionalities and technological advantages. Nanotechnol. Rev. 2015, 4, 595-624. [CrossRef]

4. Krishnan, K.M.; Pakhomov, A.B.; Bao, Y.; Blomqvist, P.; Chun, Y.; Gonzales, M.; Griffin, K.; Ji, X.; Roberts, B.K. Nanomagnetism and spin electronics: Materials, microstructure and novel properties. J. Mater. Sci. 2006, 41, 793-815. [CrossRef]

5. Ge, J.; Hu, Y.; Biasini, M.; Beyermann, W.P.; Yin, Y. Superparamagnetic magnetite colloidal nanocrystal clusters. Angew. Chem. Int. Ed. 2007, 46, 4342-4345. [CrossRef]

6. Kostopoulou, A.; Tsiaoussis, I.; Lappas, A. Magnetic iron oxide nanoclusters with tunable optical response. Photonics Nanostruct. Fundam. Appl. 2011, 9, 201-206. [CrossRef]

7. Zhuang, J.; Wu, H.; Yang, Y.; Cao, Y. Controlling colloidal superparticle growth through solvophobic interactions. Angew. Chem. Int. Ed. 2008, 47, 2208-2212. [CrossRef]

8. Ge, J.P.; Hu, Y.X.; Biasini, M.; Dong, C.L.; Guo, J.H.; Beyermann, W.P.; Yin, Y.D. One-step synthesis of highly water-soluble magnetite colloidal nanocrystals. Chem. Eur. J. 2007, 13, 7153-7161. [CrossRef]

9. Ge, J.; Hu, Y.; Yin, Y. Highly tunable superparamagnetic colloidal photonic crystals. Angew. Chem. Int. Ed. 2007, 46, 7428-7431. [CrossRef]

10. Xuan, S.; Wang, Y.; Yu, J.; Leung, K.C.F. Tuning the grain size and particle size of superparamagnetic $\mathrm{Fe}_{3} \mathrm{O}_{4}$ microparticles. Chem. Mater. 2009, 21, 5079-5087. [CrossRef]

11. Gao, J.; Ran, X.; Shi, C.; Cheng, H.; Cheng, T.; Su, Y. One-step solvothermal synthesis of highly water-soluble, negatively charged superparamagnetic $\mathrm{Fe}_{3} \mathrm{O}_{4}$ colloidal nanocrystal clusters. Nanoscale 2013, 5, 7026-7033. [CrossRef] [PubMed]

12. Li, S.; Zhang, T.; Tang, R.; Qiu, H.; Wang, C.; Zhou, Z. Solvothermal synthesis and characterization of monodisperse superparamagnetic iron oxide nanoparticles. J. Magn. Magn. Mater. 2015, 379, 226-231. [CrossRef]

13. Wang, W.; Tang, B.; Wu, S.; Gao, Z.; Ju, B.; Teng, X.; Zhang, S. Controllable 5-sulfosalicylic acid assisted solvothermal synthesis of monodispersed superparamagnetic $\mathrm{Fe}_{3} \mathrm{O}_{4}$ nanoclusters with tunable size. J. Magn. Magn. Mater. 2017, 423, 111-117. [CrossRef]

14. Kim, J.; Tran, V.T.; Oh, S.; Kim, C.S.; Hong, J.C.; Kim, S.; Joo, Y.S.; Mun, S.; Kim, M.H.; Jung, J.W.; et al. scalable solvothermal synthesis of superparamagnetic $\mathrm{Fe}_{3} \mathrm{O}_{4}$ nanoclusters for bioseparation and theragnostic probes. ACS Appl. Mater. Interfaces 2018, 10, 41935-41946. [CrossRef] [PubMed]

15. Liang, J.; Ma, H.; Luo, W.; Wang, S. Synthesis of magnetite submicrospheres with tunable size and superparamagnetism by a facile polyol process. Mater. Chem. Phys. 2013, 139, 383-388. [CrossRef]

16. Luo, B.; Xu, S.; Luo, A.; Wang, W.; Wang, S.; Guo, J.; Lin, Y.; Zhao, D.; Wang, C. Mesoporous biocompatible and acid-degradable magnetic colloidal nanocrystal clusters with sustainable stability and high hydrophobic drug loading capacity. ACS Nano 2011, 5, 1428-1435. [CrossRef] [PubMed]

17. Nikitin, A.A.; Shchetinin, I.V.; Tabachkova, N.Y.; Soldatov, M.A.; Soldatov, A.V.; Sviridenkova, N.V.; Beloglazkina, E.K.; Savchenko, A.G.; Fedorova, N.D.; Abakumov, M.A.; et al. Synthesis of iron oxide nanoclusters by thermal decomposition. Langmuir 2018, 34, 4640-4650. [CrossRef] [PubMed]

18. Jia, J.; Yu, J.; Zhu, X.; Chan, K.; Wang, Y. Ultra-fast method to synthesize mesoporous magnetite nanoclusters as highly sensitive magnetic resonance probe. J. Colloid Interface Sci. 2012, 379, 1-7. [CrossRef] [PubMed]

19. Fu, J.; He, L.; Xu, W.; Zhuang, J.; Yang, X.; Zhang, X.; Wu, M.; Yin, Y. Formation of colloidal nanocrystal clusters of iron oxide by controlled ligand stripping. Chem. Commun. 2016, 52, 128-131. [CrossRef]

20. Zhuang, J.; Wu, H.; Yang, Y.; Cao, Y. Supercrystalline colloidal particles from artificial atoms. J. Am. Chem. Soc. 2007, 129, 14166-14167. [CrossRef]

21. Ninjbadgar, T.; Brougham, D.F. Epoxy ring opening phase transfer as a general route to water dispersible superparamagnetic $\mathrm{Fe}_{3} \mathrm{O}_{4}$ nanoparticles and their application as positive MRI contrast agents. Adv. Funct. Mater. 2011, 21, 4769-4775. [CrossRef] 
22. Sherwood, J.; Rich, M.; Lovas, K.; Warram, J.; Bolding, M.S.; Bao, Y. T 1 -Enhanced MRI-visible nanoclusters for imaging-guided drug delivery. Nanoscale 2017, 9, 11785-11792. [CrossRef] [PubMed]

23. Li, X.; Wei, Z.; Lv, H.; Wu, L.; Cui, Y.; Yao, H.; Li, J.; Zhang, H.; Yang, B.; Jiang, J. Iron oxide nanoparticles promote the migration of mesenchymal stem cells to injury sites. Int. J. Nanomed. 2019, 14, 573-589. [CrossRef]

24. Paquet, C.; de Haan, H.W.; Leek, D.; Lin, H.; Xiang, B.; Tian, G.H.; Kell, A.; Simard, B. Clusters of superparamagnetic iron oxide nanoparticles encapsulated in a hydrogel: A particle architecture generating a synergistic enhancement of the $\mathrm{T}_{2}$ relaxation. ACS Nano 2011, 5, 3104-3112. [CrossRef] [PubMed]

25. Xie, X.; Zhang, C. Controllable assembly of sydrophobic superparamagnetic iron oxide nanoparticle with mPEG-PLA copolymer and its effect on MR transverse relaxation rate. J. Nanomater. 2011. [CrossRef]

26. Li, L.; Choo, E.S.G.; Yi, J.; Ding, J.; Tang, X.; Xue, J. Superparamagnetic silica composite nanospheres (SSCNs) with ultrahigh loading of iron oxide nanoparticles via an oil-in-DEG microemulsion route. Chem. Mater. 2008, 20, 6292-6294. [CrossRef]

27. Alford, A.; Rich, M.; Kozlovskaya, V.; Chen, J.; Sherwood, J.; Bolding, M.; Warram, J.; Bao, Y.; Kharlampieva, E. Ultrasound-triggered delivery of anticancer therapeutics from MRI-visible multilayer microcapsules. Adv. Therap. 2018, 1800051. [CrossRef]

28. Maity, D.; Chandrasekharan, P.; Pradhan, P.; Chuang, K.; Xue, J.; Feng, S.; Ding, J. Novel synthesis of superparamagnetic magnetite nanoclusters for biomedical applications. J. Mater. Chem. 2011, 21, 14717-14724. [CrossRef]

29. Zhang, H.; Wu, J.; Wang, X.; Li, X.; Wu, M.; Liang, F.; Yang, Y. One-pot solvothermal synthesis of Carboxylatopillar 5 arene-modified $\mathrm{Fe}_{3} \mathrm{O}_{4}$ magnetic nanoparticles for ultrafast separation of cationic dyes. Dyes Pigm. 2019, 162, 512-516. [CrossRef]

30. Li, M.; Gu, H.; Zhang, C. Highly sensitive magnetite nano clusters for MR cell imaging. Nanoscale Res. Lett. 2012, 7, 204. [CrossRef]

31. Yang, P.; Li, H.; Zhang, S.; Chen, L.; Zhou, H.; Tang, R.; Zhou, T.; Bao, F.; Zhang, Q.; He, L.; et al. Gram-scale synthesis of superparamagnetic $\mathrm{Fe}_{3} \mathrm{O}_{4}$ nanocrystal clusters with long-term charge stability for highly stable magnetically responsive photonic crystals. Nanoscale 2016, 8, 19036-19042. [CrossRef] [PubMed]

32. Borlido, L.; Azevedo, A.M.; Roque, A.C.A.; Aires-Barros, M.R. Magnetic separations in biotechnology. Biotechnol. Adv. 2013, 31, 1374-1385. [CrossRef] [PubMed]

33. Ditsch, A.; Lindenmann, S.; Laibinis, P.E.; Wang, D.I.C.; Hatton, T.A. High-gradient magnetic separation of magnetic nanoclusters. Ind. Eng. Chem. Res. 2005, 44, 6824-6836. [CrossRef]

34. Ezzaier, H.; Marins, J.A.; Claudet, C.; Hemery, G.; Sandre, O.; Kuzhir, P. Kinetics of aggregation and magnetic separation of multicore iron oxide nanoparticles: Effect of the grafted layer thickness. Nanomaterials 2018, 8 , 623. [CrossRef] [PubMed]

35. Zhang, J.; Zhu, M.; Yang, Y.; Cao, J.; Shi, F. Extraction of genomic DNA via superparamagnetic $\mathrm{Fe}_{3} \mathrm{O}_{4}$ magnetic colloidal nanocrystal clusters. J. Nanosci. Nanotechnol. 2018, 18, 8105-8110. [CrossRef] [PubMed]

36. Meerod, S.; Deepuppha, N.; Rutnakornpituk, B.; Rutnakornpituk, M. Reusable magnetic nanocluster coated with poly (acrylic acid) and its adsorption with an antibody and an antigen. J. Appl. Polym. Sci. 2018, 135, 46160. [CrossRef]

37. Long, X.; Li, J.; Sheng, D.; Lian, H. Low-cost iron oxide magnetic nanoclusters affinity probe for the enrichment of endogenous phosphopeptides in human saliva. RSC Adv. 2016, 6, 96210-96222. [CrossRef]

38. Wen, C.; Jiang, Y.; Li, X.; Tang, M.; Wu, L.; Hu, J.; Pang, D.; Zeng, J. Efficient enrichment and analyses of bacteria at ultralow concentration with quick-response magnetic nanospheres. ACS Appl. Mater. Interfaces 2017, 9, 9416-9425. [CrossRef]

39. Kim, Y.T.; Kim, K.H.; Kang, E.S.; Jo, G.; Ahn, S.Y.; Park, S.H.; Kim, S.I.; Mun, S.; Baek, K.; Kim, B.; et al. Synergistic effect of detection and separation for pathogen using magnetic clusters. Bioconjug. Chem. 2016, 27, 59-65. [CrossRef]

40. Ma, W.; Sha, X.; Gao, L.; Cheng, Z.; Meng, F.; Cai, J.; Tan, D.; Wang, R. Effect of iron oxide nanocluster on enhanced removal of molybdate from surface water and pilot scale test. Colloids Surf. A Physicochem. Eng. Asp. 2015, 478, 45-53. [CrossRef]

41. Lee, S.H.; Cha, J.; Sim, K.; Lee, J.K. Efficient removal of arsenic using magnetic multi-granule nanoclusters. Bull. Korean Chem. Soc. 2014, 35, 605-609. [CrossRef] 
42. Sherwood, J.; Sowell, J.; Beyer, N.; Irvin, J.; Stephen, C.; Antone, A.J.; Bao, Y.P.; Ciesla, L.M. Cell-membrane coated iron oxide nanoparticles for isolation and specific identification of drug leads from complex matrices. Nanoscale 2019, 11, 6352-6359. [CrossRef]

43. Bu, Y.; Hu, Q.; Ke, R.; Sui, Y.; Xie, X.; Wang, S. Cell membrane camouflaged magnetic nanoparticles as a biomimetic drug discovery platform. Chem. Comm. 2018, 54, 13427-13430. [CrossRef]

44. Hu, Q.; Bu, Y.; Zhen, X.; Xu, K.; Ke, R.; Xie, X.; Wang, S. Magnetic carbon nanotubes camouflaged with cell membrane as a drug discovery platform for selective extraction of bioactive compounds from natural products. Chem. Eng. J. 2019, 364, 269-279. [CrossRef]

45. Chen, H.; Fang, Z.; Chen, Y.; Chen, Y.; Yao, B.; Cheng, J.; Chien, C.; Chang, Y.; Hu, C. Targeting and enrichment of viral pathogen by cell membrane cloaked magnetic nanoparticles for enhanced detection. ACS Appl. Mater. Interfaces 2017, 9, 39953-39961. [CrossRef]

46. Zhang, Y.; Li, L.; Ma, W.; Zhang, Y.; Yu, M.; Guo, J.; Lu, H.; Wang, C. Two-in-one strategy for effective enrichment of phosphopeptides using magnetic mesoporous gamma- $\mathrm{Fe}_{2} \mathrm{O}_{3}$ nanocrystal clusters. ACS Appl. Mater. Interfaces 2013, 5, 614-621. [CrossRef]

47. Yang, Q.; Lan, F.; Yi, Q.; Wu, Y.; Gu, Z. A colloidal assembly approach to synthesize magnetic porous composite nanoclusters for efficient protein adsorption. Nanoscale 2015, 7, 17617-17622. [CrossRef]

48. Bueno, P.V.A.; Hilamatu, K.C.P.; Carmona-Ribeiro, A.M.; Petri, D.F.S. Magnetically triggered release of amoxicillin from xanthan/Fe3O4/albumin patches. Int. J. Biol. Macromol. 2018, 115, 792-800. [CrossRef]

49. Kuo, C.; Liu, T.; Wang, K.; Hardiansyah, A.; Lin, Y.; Chen, H.; Chiu, W.Y. Magnetic and thermal-sensitive poly(N-isopropylacrylamide)-based microgels for magnetically triggered Controlled release. J. Vis. Exp. 2017. [CrossRef]

50. Benyettou, F.; Flores, J.A.O.; Ravaux, F.; Rezgui, R.; Jouiad, M.; Nehme, S.I.; Parsapur, R.K.; Olsen, J.C.; Selvam, P.; Trabolsi, A. Mesoporous gamma-iron oxide nanoparticles for magnetically triggered release of doxorubicin and hyperthermia treatment. Chem. Eur. J. 2016, 22, 17018-17026. [CrossRef]

51. Hua, X.; Yang, Q.; Dong, Z.; Zhang, J.; Zhang, W.; Wang, Q.; Tan, S.; Smyth, H.D.C. Magnetically triggered drug release from nanoparticles and its applications in anti-tumor treatment. Drug Deliv. 2017, 24, 511-518. [CrossRef]

52. Xu, F.; Cheng, C.; Chen, D.; Gu, H. Magnetite nanocrystal clusters with ultra-high sensitivity in magnetic resonance imaging. ChemPhysChem 2012, 13, 336-341. [CrossRef]

53. Smith, C.E.; Ernenwein, D.; Shkumatov, A.; Clay, N.E.; Lee, J.Y.; Melhem, M.; Misra, S.; Zimmerman, S.C.; Kong, H. Hydrophilic packaging of iron oxide nanoclusters for highly sensitive imaging. Biomaterials 2015, 69, 184-190. [CrossRef]

54. Wu, M.; Zhang, D.; Liu, X. Nanoclusters of superparamagnetic iron oxide nanoparticles coated with poly(dopamine) for magnetic field-directed, ultrasensitive MRI-guided photothermal cancer therapy. J. Control. Release 2015, 213, 78. [CrossRef]

55. Carregal-Romero, S.; Guardia, P.; Yu, X.; Hartmann, R.; Pellegrino, T.; Parak, W.J. Magnetically triggered release of molecular cargo from iron oxide nanoparticle loaded microcapsules. Nanoscale 2015, 7, 570-576. [CrossRef]

56. Dong, F.; Guo, W.; Bae, J.; Kim, S.H.; Ha, C. Highly porous, water-soluble, superparamagnetic, and biocompatible magnetite nanocrystal clusters for targeted drug delivery. Chem. Eur. J. 2011, 17, 12802-12808. [CrossRef]

57. Lai, P.; Huang, R.; Lin, S.; Lin, Y.; Chang, C. Biomimetic stem cell membrane-camouflaged iron oxide nanoparticles for theranostic applications. RSC Adv. 2015, 5, 98222-98230. [CrossRef]

58. Meng, Q.; Rao, L.; Zan, M.; Chen, M.; Yu, G.; Wei, X.; Wu, Z.; Sun, Y.; Guo, S.; Zhao, X.; et al. Macrophage membrane-coated iron oxide nanoparticles for enhanced photothermal tumor therapy. Nanotechnology 2018, 29. [CrossRef]

59. Yu, G.; Rao, L.; Wu, H.; Yang, L.; Bu, L.; Deng, W.; Wu, L.; Nan, X.; Zhang, W.; Zhao, X.; et al. Myeloid-derived suppressor cell membrane-coated magnetic nanoparticles for cancer theranostics by Inducing macrophage polarization and synergizing immunogenic cell death. Adv. Funct. Mater. 2018, 28, 1801389. [CrossRef]

60. Bu, L.; Rao, L.; Yu, G.; Chen, L.; Deng, W.; Liu, J.; Wu, H.; Meng, Q.; Guo, S.; Zhao, X.; et al. Cancer stem cell-platelet hybrid membrane-coated magnetic nanoparticles for enhanced photothermal therapy of head and neck squamous cell carcinoma. Adv. Funct. Mater. 2019, 29. [CrossRef] 
61. Bao, Y.; Sherwood, J.A.; Sun, Z. Magnetic iron oxide nanoparticles as $\mathrm{T}_{1}$ contrast agents for magnetic resonance imaging. J. Mater. Chem. C 2018, 6, 1280-1290. [CrossRef]

62. Lu, Y.; Xu, Y.; Zhang, G.; Ling, D.; Wang, M.; Zhou, Y.; Wu, Y.; Wu, T.; Hackett, M.J.; Hyo Kim, B.; et al. Iron oxide nanoclusters for T1 magnetic resonance imaging of non-human primates. Nat. Biomed. Eng. 2017, 1, 637-643. [CrossRef]

63. Macher, T.; Totenhagen, J.; Sherwood, J.; Qin, Y.; Gurler, D.; Bolding, M.S.; Bao, Y. Ultrathin iron oxide nanowhiskers as positive contrast agents for magnetic resonance imaging. Adv. Funct. Mater. 2015, 25, 490-494. [CrossRef]

64. Sherwood, J.; Lovas, K.; Rich, M.; Yin, Q.; Lackey, K.; Bolding, M.S.; Bao, Y. Shape-dependent cellular behaviors and relaxivity of iron oxide-based T-1 MRI contrast agents. Nanoscale 2016, 8, 17506-17515. [CrossRef]

65. Kostopoulou, A.; Brintakis, K.; Fragogeorgi, E.; Anthousi, A.; Manna, L.; Begin-Colin, S.; Billotey, C.; Ranella, A.; Loudos, G.; Athanassakis, I.; et al. Iron oxide colloidal nanoclusters as theranostic vehicles and their interactions at the cellular level. Nanomaterials 2018, 8, 315. [CrossRef]

66. Lartigue, L.; Hugounenq, P.; Alloyeau, D.; Clarke, S.P.; Levy, M.; Bacri, J.C.; Bazzi, R.; Brougham, D.F.; Wilhelm, C.; Gazeau, F. Cooperative organization in iron oxide multi-core nanoparticles potentiates their efficiency as heating mediators and MRI contrast agents. ACS Nano 2012, 6, 10935-10949. [CrossRef]

67. Tang, Y.; Liu, Y.; Li, W.; Xie, Y.; Li, Y.; Wu, J.; Wang, S.; Tian, Y.; Tian, W.; Teng, Z.; et al. Synthesis of sub-100 nm biocompatible superparamagnetic $\mathrm{Fe}_{3} \mathrm{O}_{4}$ colloidal nanocrystal clusters as contrast agents for magnetic resonance imaging. RSC Adv. 2016, 6, 62550-62555. [CrossRef]

(C) 2019 by the authors. Licensee MDPI, Basel, Switzerland. This article is an open access article distributed under the terms and conditions of the Creative Commons Attribution (CC BY) license (http://creativecommons.org/licenses/by/4.0/). 